\title{
Distributed Cubature Kalman-Probability Hypothesis Density Filter for Multiple Targets Tracking
}

\author{
Liming Chen ${ }^{1 *}$ Mingxin Jiang ${ }^{2}$ and Chuanping Tong ${ }^{3}$ \\ ${ }^{1}$ College of Information and Communication Engineering, Dalian Minzu \\ University, Dalian, 116600, China \\ ${ }^{2}$ Faculty of Computer and Software Engineering, Huaiyin Institute of Technology, \\ Huaiyin, 223001, China \\ ${ }^{3}$ Dalian Air Force Communication Sergeant College, Dalian, 116600, China \\ ${ }^{1}$ chenliming@dlnu.edu.cn, ${ }^{2}$ jmx @dlnu.edu.cn, ${ }^{3}$ tongchuanping@163.com
}

\begin{abstract}
Aiming at nonlinear system model in distributed multi-target tracking, a distributed cubature Kalman-probability hypothesis density filter is proposed for multiple targets tracking in this paper. A group of sensors are used to provide measurements in the filter, third-degree spherical-radial rule is utilized to compute the multidimensional integral in Bayesian filter of nonlinear system, tags are set for every Gaussian component to distinguish targets after probability hypothesis density recursion in local estimation, and global estimation of targets' states are finally obtained by a fusion algorithm that uses local estimation of all nodes in the network. In simulation experiments, compared with the results of extended Kalman probability hypothesis density (EK-PHD) filter, unscented Kalman probability hypothesis density (UK-PHD) filter and cubature Kalman probability hypothesis density (CK-PHD) filter, the robustness of proposed algorithm is enhanced, and estimation accuracy of multiple targets number and states is improved.
\end{abstract}

Keywords: Multiple targets tracking, Probability hypothesis density filter, Kalman filter, Nonlinear filter

\section{Introduction}

Multi-target tracking is to estimate moving targets' states by signal processing algorithm. It has two kinds of approaches according to different sensor numbers it uses: single sensor and sensor network. As more information can be collected with multiple sensors, tracking algorithm working on a sensor network is adopted in many fields, such as video surveillance, disaster response, environmental monitoring, etc. [1]. According to different information fusion approaches, multi-target tracking in a sensor network can be grossly divided into three groups: centralized, hierarchical and distributed schemes [2]. In this paper, we focus on distributed multi-target tracking schemes.

\subsection{Single Sensor Multi-Target Tracking}

Choosing appropriate single sensor multi-target tracking algorithm is essential to distributed multi-target tracking. When mapping single sensor multi-target tracking algorithm onto a sensor network, collaborative information processing method is utilized in distributed multi-target tracking [3].

Most traditional multi-target tracking approaches have a common drawback that their computational complexity grows rapidly when the number of measurements increases because they apply data association techniques, such as global nearest neighbor (GNN),

Received (November 28, 2016), Review Result (October 18, 2017), Accepted (October 31, 2017) 
joint probabilistic data association (JPDA), and multiple hypothesis tracking (MHT) [4].

To remedy the problems above, Mahler proposed random finite set (RFS) theory to track multiple targets [5]. The probability hypothesis density (PHD) filter [6], as a kind of RFS-based filter, is a recursion form of posterior intensity, which is a first-order statistical moment of posterior multi-target states. According to different system model, PHD filter can be divided into linear and nonlinear system. Available multi-target tracking algorithms at present of nonlinear system has two categories, i.e. global approaches and local approaches [7].

(1) Global approaches: These approaches do not make any explicit assumptions about the posterior density form, but have high computational complexity, for example, Gaussian-sum probability hypothesis density (GSPHD) filter [8] and sequential Monte Carlo-probability hypothesis density (SMC-PHD) filter [9], etc..

(2) Local approaches: Although these approaches can only be applied to specific circumstances because they need a priori form to posterior density, they have lower computational complexity than global approaches and simpler algorithm which makes them easier when implementing, for example, extended Kalman probability hypothesis density (EK-PHD) filter [10], unscented Kalman probability hypothesis density (UK-PHD) filter [10], central difference Kalman probability hypothesis density (CDK-PHD) filter [11-12], cubature Kalman probability hypothesis density (CK-PHD) filter [13].

\subsection{Distributed Target Tracking}

Distributed estimation and tracking is initially used in single target tracking problem [14]. Focused on linear system, Olfati-Saber proposed distributed Kalman filter (DKF) which applies dynamic consensus algorithms in micro-Kalman filters to a sensor network [15]. Aiming at non-linear system, distributed version of unscented Kalman filter [16] and distributed particle filter (DPF) [14] have been developed recently.

In distributed multi-target tracking field, inspired by DPF, DPF with Gaussian Mixer approximation algorithm is proposed to localize and track multiple moving targets in [17]. Another distributed multi-target tracking algorithm using joint probabilistic data association (JPDA) and average consensus filter which points at nonlinear and nonGaussian system models is proposed recently [6]. Those algorithms both have limitations on targets' number. The former method requires that the number is pre-known and the latter needs it to be fixed [6].

\subsection{Main Idea of Proposed Algorithm}

The motivation of this paper is to build a nonlinear filter which is more accurate and robust, with fewer limitations, and could be applicable to multi-target tracking problem in a sensor network. Aiming at nonlinear Gaussian system model, we take CK-PHD filter as multi-target tracking method to obtain local estimation of every node. We added tag algorithm to CK-PHD filter to discriminate different targets so that CK-PHD could be mapped to a sensor network. Finally, a fusion algorithm is taken to generate a global estimation of every target.

Theoretically, the proposed algorithm combines the advantages of CK-PHD, distributed multi-target tracking and tag algorithm. It can be more robust even some sensor nodes have detection problems, can enhance the precision of estimation of targets' and positions, has lower computational complexity when number of measurements increases, has lower network band width demand, and has no requirement to targets' number information. Simulation results have proved the validity of proposed method.

This paper is organized as follows. Section II describes nonlinear system model of multi-target tracking, and summarizes CK-PHD filter. Section III presents distributed CKPHD filter for multi-target tracking. In section IV, effects of proposed approach are demonstrated via simulations. Finally, conclusions are given in section V. 


\section{Cubature Kalman-Probability Hypothesis Density Filter [13]}

Let $n_{x}$-dimensional vector $\boldsymbol{x}_{k}$ denote target state, $n_{z}$-dimensional vector $\boldsymbol{z}_{k}$ denote target measurement, then nonlinear dynamic system model [1] is defined by

$$
\begin{gathered}
\boldsymbol{x}_{k}=f_{k}\left(\boldsymbol{x}_{k-1}, \boldsymbol{u}_{k-1}\right)+\boldsymbol{v}_{k-1} \\
\boldsymbol{z}_{k}=g_{k}\left(\boldsymbol{x}_{k}, \boldsymbol{u}_{k}\right)+\boldsymbol{w}_{k}
\end{gathered}
$$

$f_{k}$ and $g_{k}$ are both nonlinear functions, $\boldsymbol{u}_{k}$ is control input, $\boldsymbol{v}_{k-1}$ and $\boldsymbol{w}_{k}$ are $n_{v}-$ and $n_{w}$ - dimensional i.i.d. white Gaussian noise respectively, and satisfied with $\boldsymbol{v}_{k-1} \sim \mathrm{N}\left(\mathbf{0}, \boldsymbol{Q}_{k-1}\right), \boldsymbol{w}_{k} \sim \mathrm{N}\left(\mathbf{0}, \boldsymbol{R}_{k}\right)$.

CK-PHD filter, which does not need to calculate Jacobian matrix or to set scaling parameters, is a kind of implementation form for PHD filter. Generally, CK-PHD filter that uses nonlinear system model (1)-(2) has three main steps, i.e., the PHD recursion, the pruning and the state extraction [13]. The PHD recursion is the key step of the filter and comprises two parts, prediction and update.

\subsection{Prediction of PHD Recursion}

In the prediction part of PHD recursion, predicted intensity $v_{k \mid k-1}(\boldsymbol{x})[10]$ is given by

$$
v_{k \mid k-1}(\boldsymbol{x})=v_{S, k \mid k-1}(\boldsymbol{x})+v_{\beta, k \mid k-1}(\boldsymbol{x})+\gamma_{k}(\boldsymbol{x})
$$

where $v_{S, k \mid k-1}(\boldsymbol{x}), v_{\beta, k \mid k-1}(\boldsymbol{x})$, and $\gamma_{k}(\boldsymbol{x})$ denote surviving intensity, spawning intensity, and spontaneous birth intensity respectively. Each of them can be expressed by Gaussian sum [10], i.e.

$$
\begin{gathered}
v_{S, k \mid k-1}(\boldsymbol{x})=\sum_{j=1}^{J_{k-1}} w_{S, k \mid k-1}^{(j)} \mathrm{N}\left(\boldsymbol{x} ; \boldsymbol{m}_{S, k \mid k-1}^{(j)}, \boldsymbol{P}_{S, k \mid k-1}^{(j)}\right) \\
v_{\beta, k \mid k-1}(\boldsymbol{x})=\sum_{j=1}^{J_{k-1}} \sum_{l=1}^{J_{\beta, k}} w_{\beta, k \mid k-1}^{(j, l)} \mathrm{N}\left(\boldsymbol{x} ; \boldsymbol{m}_{\beta, k \mid k-1}^{(j, l)}, \boldsymbol{P}_{\beta, k \mid k-1}^{(j, l)}\right) \\
\gamma_{k}(\boldsymbol{x})=\sum_{j=1}^{J_{\gamma, k}} w_{\gamma, k}^{(j)} \mathrm{N}\left(\boldsymbol{x} ; \boldsymbol{m}_{\gamma, k}^{(j)}, \boldsymbol{P}_{\gamma, k}^{(j)}\right)
\end{gathered}
$$

where $J_{k-1}, w_{S, k \mid k-1}^{(j)}, \boldsymbol{m}_{S, k \mid k-1}^{(j)}, \boldsymbol{P}_{S, k \mid k-1}^{(j)}, j=1, \cdots, J_{k-1}$ are the number, weights, means, and covariances of Gaussian components in surviving intensity $v_{S, k \mid k-1}(\boldsymbol{x})$. Suppose that each target in time $k-1$ may spawn $J_{\beta, k}$ targets. Then $w_{\beta, k}^{(l)}, \boldsymbol{m}_{\beta, k \mid k-1}^{(j, l)}, \boldsymbol{P}_{\beta, k \mid k-1}^{(j, l)}$, $j=1, \cdots, J_{k-1}, l=1, \cdots, J_{\beta, k}$ are the weights, means, and covariance of Gaussian components in spawning intensity $v_{\beta, k \mid k-1}(\boldsymbol{x})$, whose parameters are described in [10]. In equation (6), $J_{\gamma, k}, w_{\gamma, k}^{(j)}, m_{\gamma, k}^{(j)}, P_{\gamma, k}^{(j)}, j=1, \cdots, J_{\gamma, k}$, parameters of spontaneous birth intensity $\gamma_{k}(x)$, are set as input of filter. Section IV presents parameters' values of $\gamma_{k}(x)$ in simulation.

Let $J_{k-1}, w_{k-1}^{(j)}, \boldsymbol{m}_{k-1}^{(j)}, \boldsymbol{P}_{k-1}^{(j)}$ denote the number, weight, mean, and covariance of the $j$ th Gaussian component in the intensity $v_{k-1}$ at time $k-1$ respectively, where $j=1,2, \cdots, J_{k-1}$. Therefore the weight, mean, and covariance of Gaussian components in surviving intensity $v_{S, k \mid k-1}(\boldsymbol{x})$ are given in $[7,13]$. 
Let $J_{k \mid k-1}$ denote the number of Gaussian components for predicted intensity $v_{k \mid k-1}(\boldsymbol{x})$. $v_{k \mid k-1}(\boldsymbol{x})$ is given by

$$
v_{k \mid k-1}(\boldsymbol{x})=\sum_{i=1}^{J_{k \mid k-1}} w_{k \mid k-1}^{(i)} \mathrm{N}\left(\boldsymbol{x} ; \boldsymbol{m}_{k \mid k-1}^{(i)}, \boldsymbol{P}_{k \mid k-1}^{(i)}\right)
$$

where $w_{k \mid k-1}^{(i)}, \boldsymbol{m}_{k \mid k-1}^{(i)}, \boldsymbol{P}_{k \mid k-1}^{(i)}, i=1,2, \ldots, J_{k \mid k-1}$ are the weight, mean, and covariance of Gaussian components in $v_{k \mid k-1}(\boldsymbol{x})$.

\subsection{Update of PHD Recursion}

In the update part of PHD recursion, posterior intensity $v_{k}(\boldsymbol{x})$ at time $k$ can be expressed by a Gaussian mixture [13], i.e.,

$$
\begin{gathered}
v_{k}(\boldsymbol{x})=\left(1-p_{D, k}\right) v_{k \mid k-1}(\boldsymbol{x})+\sum_{z \in \boldsymbol{Z}_{k}} v_{D, k}(\boldsymbol{x} ; \boldsymbol{z}) \\
v_{D, k}(\boldsymbol{x} ; \boldsymbol{z})=\sum_{j=1}^{J_{k \mid k-1}} w_{k}^{(j)}(\boldsymbol{z}) \mathrm{N}\left(\boldsymbol{x} ; \boldsymbol{m}_{k \mid k}^{(j)}(\boldsymbol{z}), \boldsymbol{P}_{k \mid k}^{(j)}\right)
\end{gathered}
$$

where $p_{D, k}$ is the probability of detection at time $k, \boldsymbol{Z}_{k}$ is the measurements, $\left(1-p_{D, k}\right) v_{k \mid k-1}(\boldsymbol{x})$ denotes intensity of miss detection. Posterior intensity of detection $v_{D, k}(\boldsymbol{x} ; \boldsymbol{z})$ is a Gaussian mixture, whose weight, mean, and covariance are $w_{k}^{(j)}(\boldsymbol{z})$, $\boldsymbol{m}_{k \mid k}^{(j)}(\boldsymbol{z}), \boldsymbol{P}_{k \mid k}^{(j)}, j=1,2, \ldots, J_{k \mid k-1}$ respectively. The parameters are described in [13].

Let $J_{k}$ denote the number of Gaussian components in posterior intensity $v_{k}(\boldsymbol{x})$ at time $k$. Based on equations (7)-(9), $v_{k}(\boldsymbol{x})$ is given by

$$
v_{k}(\boldsymbol{x})=\sum_{i=1}^{J_{k}} w_{k}^{(i)} \mathrm{N}\left(\boldsymbol{x} ; \boldsymbol{m}_{k \mid k}^{(i)}, \boldsymbol{P}_{k \mid k}^{(i)}\right)
$$

where $w_{k}^{(i)}, \boldsymbol{m}_{k \mid k}^{(i)}, P_{k \mid k}^{(i)}, i=1,2, \ldots, J_{k}$ are the weight, mean, and covariance of Gaussian components in $v_{k}(\boldsymbol{x})$.

\subsection{Pruning and Merging Algorithm in CK-PHD Filter}

In implementation, the CK-PHD filter suffers from computation problem, since the number of Gaussian components in the intensity increases without bound. Pruning and merging algorithm can reduce the number of Gaussian components significantly. These algorithms discard Gaussian components with lower weights, and then merge together the components whose means are close.

\subsection{State Extraction in CK-PHD Filter}

Multiple targets' states can be obtained from the means of Gaussian components after pruning, because the means are local maxima of intensity $v_{k}$. And in order to remove some local maxima with very low peaks, only components those have weights higher than 0.5 can be used to extract targets states. 


\section{Distributed CK-PHD Filter for Multiple Targets Tracking}

\subsection{Problem Formulation}

In distributed multi-target tracking algorithms, the measurements are dispersed among nodes. Let the sensor network consist of $\boldsymbol{M}$ nodes, $\boldsymbol{Z}_{m, k}=\left\{\boldsymbol{z}_{m, k}\right\}$ is the set of local measurements for node $m \in\{1,2, \ldots, M\}$ at time k. It includes the measurements of targets and clutters which are generated by false alarm. And the number of measurements in $\boldsymbol{Z}_{m, k}$, that is $\left|\boldsymbol{Z}_{m, k}\right|$, is unknown. The local measurement model is given by

$\boldsymbol{z}_{m, k}=g_{m, k}\left(\boldsymbol{x}_{k}, \boldsymbol{u}_{k}\right)+\boldsymbol{w}_{m, k}$

where $g_{m, k}$ is nonlinear function, $\boldsymbol{w}_{m, k}$ is measurement noise for node $m$, and satisfied with $\boldsymbol{w}_{m, k} \sim \mathrm{N}\left(\mathbf{0}, \boldsymbol{R}_{m, k}\right)$. As $\boldsymbol{x}_{k}$ denotes target state, and the relation between $\boldsymbol{x}_{k}$ and $\boldsymbol{x}_{k-1}$ is unaffected by different nodes. Then, the process model is the same with global system model (1), and we assume that process model (1) and measurement model (11) are available to each node.

Our purpose, tracking every target, needs to at first estimate state for each target at each node using measurements $\boldsymbol{Z}_{m, k}$ and the prior, and then compute the final global estimation result by information fusion.

\subsection{Tag Management Algorithm}

In order to obtain targets' states, distributed multi-target tracking computes local estimation first, then acquire global estimation through an associate algorithm. Unlike single target tracking, distributed multi-target tracking needs to distinguish different targets in every node during the process of local estimation computing. In proposed method, tag management algorithm is adopted.

Tag management algorithm [29], proposed by Kusha Panta, is taken to obtain a GMPHD tracker which can track different targets in the scenario. In this paper, inspired by the document [29], tag labels are utilized in Gaussian components and propagate in the whole PHD recursion of CK-PHD. The specific procedure of using tag management algorithm in local estimation is as follows.

(1) Set tag labels for every Gaussian component: Consider the local estimation for node $m \in\{1,2, \ldots, M\}$. Tag labels are set in every Gaussian component for every node, that is, let $\left\{w_{m, k-1}^{(i)}, \boldsymbol{m}_{m, k-1}^{(i)}, \boldsymbol{P}_{m, k-1}^{(i)}, \tau_{m, k-1}^{(i)}\right\}_{i=1}^{J_{m, k-1}}$ denote Gaussian components of intensity $v_{k-1}(\boldsymbol{x})$ at time $k-1$. Here, $w_{m, k-1}^{(i)}, \boldsymbol{m}_{m, k-1}^{(i)}, \boldsymbol{P}_{m, k-1}^{(i)}$ are the weight, mean and covariance and a unique identifier of the $i$ th component. $\tau_{m, k-1}^{(i)}$ is tag label of the component, which is a unique identifier.

(2) Tag management for PHD recursion: In the prediction part, let $\left\{w_{m, \gamma, k}^{(i)}, \boldsymbol{m}_{m, \gamma, k}^{(i)}, \boldsymbol{P}_{m, \gamma, k}^{(i)}, \tau_{m, \gamma, k}^{(i)}\right\}_{i=1}^{J_{m, \gamma, k}}$ and $\left\{w_{m, \beta, k \mid k-1}^{(j, l)}, \boldsymbol{m}_{m, \beta, k \mid k-1}^{(j, l)}, \quad \boldsymbol{P}_{m, \beta, k \mid k-1}^{(j, l)}, \tau_{m, \beta, k \mid k-1}^{(j, l)}\right\}_{j=1, l=1}^{J_{m, k-1}, J_{m, \beta, k-1}}$ denote Gaussian components of $\gamma_{k}(\boldsymbol{x})$ and $v_{\beta, k \mid k-1}(\boldsymbol{x})$ for node $m$ respectively, where $w_{m, \gamma, k}^{(i)}, \boldsymbol{m}_{m, \gamma, k}^{(i)}, \boldsymbol{P}_{m, \gamma, k}^{(i)}, \tau_{m, \gamma, k}^{(i)}$ are the weight, mean, covariance and tag label of the $i$ th component of $\gamma_{k}(\boldsymbol{x}), w_{m, \beta, k \mid k-1}^{(j, l)}, \boldsymbol{m}_{m, \beta, k \mid k-1}^{(j, l)}, \boldsymbol{P}_{m, \beta, k \mid k-1}^{(j, l)}, \tau_{m, \beta, k \mid k-1}^{(j, l)}$ are the weight, mean, covariance and tag label of the component of $v_{\beta, k \mid k-1}(\boldsymbol{x})$.

According to equations (4) and (7)-(10), the components of $v_{S, k \mid k-1}(\boldsymbol{x})$ are given by 
$\left\{w_{m, S, k \mid k-1}^{(i)}, \quad \boldsymbol{m}_{m, S, k \mid k-1}^{(i)}, \boldsymbol{P}_{m, S, k \mid k-1}^{(i)}, \tau_{m, k-1}^{(i)}\right\}_{i=1}^{J_{m, k-1}}$. Consequently, using equation (3), the

Gaussian components of $v_{k \mid k-1}(\boldsymbol{x})$ for node $m$ are constructed by three parts:

$v_{k \mid k-1}(\boldsymbol{x}):\left\{w_{m, k \mid k-1}^{(i)}, \boldsymbol{m}_{m, k \mid k-1}^{(i)}, \boldsymbol{P}_{m, k \mid k-1}^{(i)}, \tau_{m, k \mid k-1}^{(i)}\right\}_{i=1}^{J_{m, k \mid k-1}}$

(1) $\gamma_{k}(\boldsymbol{x}):\left\{w_{m, \gamma, k}^{(i)}, \boldsymbol{m}_{m, \gamma, k}^{(i)}, \boldsymbol{P}_{m, \gamma, k}^{(i)}, \tau_{m, \gamma, k}^{(i)}\right\}_{i=1}^{J_{m, \gamma, k}}$

(2) $v_{\beta, k \mid k-1}(\boldsymbol{x}):\left\{w_{m, \beta, k \mid k-1}^{(j, l)}, \boldsymbol{m}_{m, \beta, k \mid k-1}^{(j, l)}, \boldsymbol{P}_{m, \beta, k \mid k-1}^{(j, l)}, \tau_{m, \beta, k \mid k-1}^{(j, l)}\right\}_{j=1, l=1}^{J_{m, k-1}, J_{m, \beta, k-1}}$

(3) $v_{S, k \mid k-1}(\boldsymbol{x}):\left\{w_{m, S, k \mid k-1}^{(i)}, \boldsymbol{m}_{m, S, k \mid k-1}^{(i)}, \boldsymbol{P}_{m, S, k \mid k-1}^{(i)}, \tau_{m, k-1}^{(i)}\right\}_{i=1}^{J_{m, k-1}}$

where $w_{m, k \mid k-1}^{(i)}, \boldsymbol{m}_{m, k \mid k-1}^{(i)}, \boldsymbol{P}_{m, k \mid k-1}^{(i)}, \tau_{m, k \mid k-1}^{(i)}$ are the weight, mean, covariance and tag label of the $i$ th component of $v_{k \mid k-1}(\boldsymbol{x})$.

In the update part, according to (8)-(9), posterior intensity $v_{k}(\boldsymbol{x})$ for node $m$ can be expressed by a Gaussian mixture. The number of Gaussian components $J_{m, k}$ is given by

$$
J_{m, k}=J_{m, k \mid k-1}+\left|Z_{m, k}\right| J_{m, k \mid k-1}
$$

where $J_{m, k \mid k-1}$ is components number of $v_{k \mid k-1}(\boldsymbol{x})$ for node $m,\left|\boldsymbol{Z}_{m, k}\right|$ is the number of measurement set $\boldsymbol{Z}_{m, k}$.

Let $\left\{w_{m, k}^{(i)}, \boldsymbol{m}_{m, k}^{(i)}, \boldsymbol{P}_{m, k}^{(i)}, \tau_{m, k}^{(i)}\right\}_{i=1}^{J_{m, k}}$ denote Gaussian components of $v_{k}(\boldsymbol{x})$ for node $m$, where $w_{m, k}^{(i)}, \boldsymbol{m}_{m, k}^{(i)}, \boldsymbol{P}_{m, k}^{(i)}, \tau_{m, k}^{(i)}$ are the weight, mean, covariance and tag label of the $i$ th component of $v_{k}(\boldsymbol{x})$. The tag labels of $v_{k}(\boldsymbol{x})$ for node $m$ are the same as its associated predict term for every $\boldsymbol{z} \in \boldsymbol{Z}_{m, k}$. Then, using equation (8), $v_{k}(\boldsymbol{x})$ is constructed by two parts:

$v_{k}(\boldsymbol{x}):\left\{w_{m, k}^{(i)}, \boldsymbol{m}_{m, k}^{(i)}, \boldsymbol{P}_{m, k}^{(i)}, \tau_{m, k}^{(i)}\right\}_{i=1}^{J_{m, k}}$

(1) $\left(1-p_{D, k}\right) v_{k \mid k-1}(\boldsymbol{x}):\left\{\left(1-p_{D, k}\right) w_{m, k \mid k-1}^{(i)}, \boldsymbol{m}_{m, k \mid k-1}^{(i)}, \boldsymbol{P}_{m, k \mid k-1}^{(i)} \tau_{m, k \mid k-1}^{(i)}\right\}_{i=1}^{J_{m, k \mid k-1}}$

(2) $\sum_{z \in \boldsymbol{Z}_{k}} v_{D, k}(\boldsymbol{x} ; \boldsymbol{z}):$ for $\boldsymbol{z} \in \boldsymbol{Z}_{m, k}\left\{w_{m, k}^{(i)}(\boldsymbol{z}), \boldsymbol{m}_{m, k \mid k}^{(i)}(\boldsymbol{z}), \boldsymbol{P}_{m, k \mid k}^{(i)}(\boldsymbol{z}), \tau_{m, k \mid k-1}^{(i)}\right\}_{i=1}^{J_{m, k \mid k-1}}$

where $w_{m, k}^{(i)}(\boldsymbol{z}), \boldsymbol{m}_{m, k \mid k}^{(i)}(\boldsymbol{z}), \boldsymbol{P}_{m, k \mid k}^{(i)}(\boldsymbol{z})$ are weights, means and covariance of posterior intensity of detection $v_{D, k}(\boldsymbol{x} ; \boldsymbol{z})$.

\subsection{Pruning and Merging Algorithm in Distributed CK-PHD Filter}

Just like CK-PHD filter, in order to reduce the number of increasing Gaussian components, pruning step is required in the proposed algorithm.

(1) Pruning algorithm: Discard the components $\left\{w_{m, k}^{(i)}, \boldsymbol{m}_{m, k}^{(i)}, \boldsymbol{P}_{m, k}^{(i)}, \tau_{m, k}^{(i)}\right\}_{i=1}^{J_{m, k}}$ of $v_{k}(\boldsymbol{x})$ for every node $m \in\{1,2, \ldots, M\}$ having low weights, and set $10^{-5}$ as threshold usually [10]. Let $\left\{w_{m, k}^{(i)}, \boldsymbol{m}_{m, k}^{(i)}, \boldsymbol{P}_{m, k}^{(i)}, \tau_{m, k}^{(i)}\right\}_{i=1}^{J_{m, k, d i s}}$ denote the components after pruning algorithm, where $J_{m, k, d i s} \leq J_{m, k}$ is the number of the components. The procedure of pruning algorithm is shown below.

$$
\begin{aligned}
& \text { input }\left\{w_{m, k}^{(i)}, \boldsymbol{m}_{m, k}^{(i)}, \boldsymbol{P}_{m, k}^{(i)}, \tau_{m, k}^{(i)}\right\}_{i=1}^{J_{m, k}} \\
& \text { for } i=1, \cdots, J_{m, k} \\
& \quad \text { if } w_{m, k}^{(i)}<10^{-5}
\end{aligned}
$$




$$
\left\{w_{m, k}^{(i)}, \boldsymbol{m}_{m, k}^{(i)}, \boldsymbol{P}_{m, k}^{(i)}, \tau_{m, k}^{(i)}\right\}_{i=1}^{J_{m, k, d i s}}=\left\{w_{m, k}^{(i)}, \boldsymbol{m}_{m, k}^{(i)}, \boldsymbol{P}_{m, k}^{(i)}, \tau_{m, k}^{(i)}\right\}_{i=1}^{J_{m, k}} \backslash\left\{w_{m, k}^{(i)}, \boldsymbol{m}_{m, k}^{(i)}, \boldsymbol{P}_{m, k}^{(i)}, \tau_{m, k}^{(i)}\right\}
$$

end

end

output $\left\{w_{m, k}^{(i)}, \boldsymbol{m}_{m, k}^{(i)}, \boldsymbol{P}_{m, k}^{(i)}, \tau_{m, k}^{(i)}\right\}_{i=1}^{J_{m, k, l i s}}$.

(2) Merging algorithm: Some components in $\left\{w_{m, k}^{(i)}, \boldsymbol{m}_{m, k}^{(i)}, \boldsymbol{P}_{m, k}^{(i)}, \tau_{m, k}^{(i)}\right\}_{i=1}^{J_{m, k, d i s}}$ are so close to each other that they can be merged into one. However, when different targets with close proximity exist in this scenario, the components of these targets might be merged into one. To tackle this problem, two new components will substitute for component which has weight higher than 2. Each new component has half of previous component's weight, same means and covariance as previous component. One of the new components holds the tag of the previous component and the other is assigned a new tag. Detail of this idea is given below.

$$
\text { input }\left\{w_{m, k}^{(i)}, \boldsymbol{m}_{m, k}^{(i)}, \boldsymbol{P}_{m, k}^{(i)}, \tau_{m, k}^{(i)}\right\}_{i=1}^{J_{m, k, d i s}}
$$

Set $l=0, I=\left\{1,2, \cdots, J_{m, k, d i s}\right\}, J_{\max }$ is a maximum number of Gaussian components, $T_{\text {merge }}$ is a merging threshold.

while $I \neq \phi$

$$
\begin{aligned}
& l:=l+1, \\
& j=\arg \max _{i \in I} w_{m, k}^{(i)}, \\
& L=\left\{i \in I \mid\left(\boldsymbol{m}_{m, k}^{(i)}-\boldsymbol{m}_{m, k}^{(j)}\right)^{T}\left(\boldsymbol{P}_{m, k}^{(i)}\right)^{-1}\left(\boldsymbol{m}_{m, k}^{(i)}-\boldsymbol{m}_{m, k}^{(j)}\right) \leq T_{m e r g e}\right\}, \\
& w_{m, k, \text { merge }}^{(l)}=\sum_{i \in L} w_{m, k}^{(i)}, \\
& \boldsymbol{m}_{m, k, \text { merge }}^{(l)}=\frac{1}{w_{m, k, \text { merge }}^{(l)}} \sum_{i \in L} w_{m, k}^{(i)} \boldsymbol{m}_{m, k}^{(i)}, \\
& \boldsymbol{P}_{m, k, \text { merge }}^{(l)}=\frac{1}{w_{m, k, \text { merge }}^{(l)}} \sum_{i \in L} w_{m, k}^{(i)}\left[\boldsymbol{P}_{m, k}^{(i)}+\left(\boldsymbol{m}_{m, k, \text { merge }}^{(l)}-\boldsymbol{m}_{m, k}^{(i)}\right)\left(\boldsymbol{m}_{m, k, \text { merge }}^{(l)}-\boldsymbol{m}_{m, k}^{(i)}\right)^{T}\right], \\
& j_{m}=\arg \max _{i \in L} w_{m, k}^{(i)}, \\
& \tau_{m, k, \text { merge }}^{(l)}=\tau_{m, k}^{\left(j_{m}\right)}, \\
& I:=I \backslash L .
\end{aligned}
$$

end

Let $J_{m, k, \text { merge }}$ is the number of Gaussian components after while statement.

for $i=1,2, \cdots, J_{m, k}$

$$
\text { if } \begin{aligned}
w_{m, k, \text { merge }}^{(i)}> & 2 \\
J_{m, k, \text { merge }} & :=J_{m, k, \text { merge }}+1, \\
w_{m, k, \text { merge }}^{J_{m, k \text { merge }}} & =0.5 w_{m, k, \text { merge }}^{(i)}, \\
w_{m, k, \text { merge }}^{(i)} & =0.5 w_{m, k, \text { merge }}^{(i)}, \\
\boldsymbol{m}_{m, k, \text { merge }}^{J_{m, k \text { merge }}} & =\boldsymbol{m}_{m, k, \text { merge }}^{(i)}, \\
\boldsymbol{P}_{m, k, \text { merge }}^{J_{m, k \text { merge }}} & =\boldsymbol{P}_{m, k, \text { merge }}^{(i)}, \\
\tau_{m, k, \text { merge }}^{J_{m, \text { merge }}} & =\max \left(\tau_{m, k, \text { merge }}^{(i)}\right)_{i=1}^{J_{m, k, \text { merge }}-1}+1 .
\end{aligned}
$$

end

end 


$$
\text { output }\left\{w_{m, k, \text { merge }}^{(i)}, \boldsymbol{m}_{m, k, \text { merge }}^{(i)}, \boldsymbol{P}_{m, k, \text { merge }}^{(i)}, \tau_{m, k, \text { merge }}^{(i)}\right\}_{i=1}^{J_{m, k \text { merge }}}
$$

\subsection{State Extraction in Distributed CK-PHD Filter}

The Gaussian components $\left\{w_{m, k, \text { merge }}^{(i)}, \boldsymbol{m}_{m, k, \text { merge }}^{(i)}, \boldsymbol{P}_{m, k, \text { merge }}^{(i)}, \tau_{m, k, \text { merge }}^{(i)}\right\}_{i=1}^{J_{m, k \text { merge }}}$ after merging are used to obtain local estimation for node $m$. Basically, state extraction in distributed CK-PHD filter uses the means of components satisfied with $w_{m, k}>0.5$ to extract local targets' states. Nevertheless, the problem that different targets have same tag in extraction result still occasionally happens due to some clutter positions are so close to real targets. In this situation, estimations of these targets are replaced by a new estimation whose state is the average value of all previous targets'. By these means, local estimation of every node can be achieved and different targets can be distinguished via tags in proposed algorithm because a target has a unique tag all the time. The state extraction procedure in distributed CK-PHD filter is shown below.

$$
\begin{aligned}
& \text { input }\left\{w_{m, k, \text { merge }}^{(i)}, \boldsymbol{m}_{m, k, \text { merge }}^{(i)}, \boldsymbol{P}_{m, k, \text { merge }}^{(i)}, \tau_{m, k, \text { merge }}^{(i)}\right\}_{i=1}^{J_{m, k \text { merge }}} \\
& \text { for } i=1,2, \cdots, J_{m, k, \text { merge }} \\
& \qquad \begin{aligned}
\text { if } w_{m, k, \text { merge }}^{(i)}<0.5 \\
\qquad\left\{\boldsymbol{m}_{m, k}^{(i)}, \tau_{m, k}^{(i)}\right\}_{i=1}^{J_{m, k \text { local }}}=\left\{\boldsymbol{m}_{m, k}^{(i)}, \tau_{m, k}^{(i)}\right\}_{i=1}^{J_{m, k \text { merge }}} \backslash\left\{\boldsymbol{m}_{m, k}^{(i)}, \tau_{m, k}^{(i)}\right\} .
\end{aligned}
\end{aligned}
$$

end

end

$$
\begin{aligned}
& \text { for } i=1,2, \cdots, J_{m, k, \text { local }} \\
& \qquad \begin{array}{l}
\operatorname{comp}=\tau_{m, k}^{(i)}, \\
I_{\text {local }}=\left\{\tilde{i}=i+1, i+2, \cdots, J_{m, k, \text { local }} \mid \operatorname{comp}=\tau_{m, k}^{(\tilde{i})}\right\} .
\end{array}
\end{aligned}
$$

end

$$
\boldsymbol{m}_{m, k}=\frac{1}{\mid I_{\text {local }}} \sum_{i \in I_{\text {local }}} m_{m, k}^{(i)} .
$$

Use $\boldsymbol{m}_{m, k}$ replace $\left\{\boldsymbol{m}_{m, k}^{(i)}\right\}_{i \in I_{\text {local }}}$, and update $J_{m, k, \text { local }}:=J_{m, k, \text { local }}-\left|I_{\text {local }}\right|$.

output $\left\{\boldsymbol{m}_{m, k}^{(i)}, \tau_{m, k}^{(i)}\right\}_{i=1}^{J_{m, k, l o c a l}}$.

\subsection{Global Estimation Algorithm}

Global estimation using the data of all nodes can be more precise and robust than local estimation which uses only the information of one node. It generates each target's state by calculating average value of all nodes' local estimations. The calculation needs a computational center to accomplish and the sketch map of the network is shown in Figure 1. Suppose there are four nodes in the network. All these nodes only communicate with computational center. Take node $A$ for the example, the local estimation of this node is sent to computational center. And other nodes have the same communication situation as node $A$. Because the nodes do not transfer observation data, the network has low bandwidth burden. The procedure of global estimation algorithm is given below.

input $\left\{\boldsymbol{m}_{m, k}^{(i)}, \tau_{m, k}^{(i)}\right\}_{i=1}^{J_{m, k} \text { local }}$.

Let $\hat{X}_{k}=\phi$ denote multi-target global estimation at time $k, \tau_{k}=\phi$ denote tag label at time $k$.

for $m=1,2, \cdots, M ; i=1,2, \cdots, J_{m, k, l o c a l}$ 


$$
\begin{aligned}
& \text { comp }=\tau_{m, k}^{(i)}, \\
& I_{k}:=\left\{\boldsymbol{m}_{m, k}^{(i)}\right\}, \\
& \text { for } \tilde{m}=m+1, m+2, \cdots, M ; \tilde{i}=1,2, \cdots, J_{\tilde{m}, k, l o c a l} \\
& I_{k}:=\left\{I_{k}, \boldsymbol{m}_{\tilde{m}, k}^{(\tilde{i})} \mid \operatorname{comp}=\tau_{\tilde{m}, k}^{(\tilde{i})}\right\}, \\
& \left\{\boldsymbol{m}_{\tilde{m}, k}^{(i)}, \tau_{\tilde{m}, k}^{(i)}\right\}_{i=1}^{J_{\tilde{m}, k, g l b a b l}}:=\left\{\boldsymbol{m}_{\tilde{m}, k}^{(i)}, \tau_{\tilde{m}, k}^{(i)}\right\}_{i=1}^{J_{\tilde{m}, k, l o c a l}} \backslash\left\{\boldsymbol{m}_{\tilde{m}, k}^{(\tilde{i})}, \tau_{\tilde{m}, k}^{(\tilde{i})}\right\} . \\
& \hat{X}_{k}:=\left[\hat{X}_{k}, \frac{1}{\left|I_{k}\right|} \sum_{j=1}^{\left|I_{k}\right|} \boldsymbol{m}_{k}^{(j)}\right], \text { where } I_{k}=\left\{\boldsymbol{m}_{k}^{(j)}, j=1, \cdots,\left|I_{k}\right|\right\}, \\
& \tau_{k}:=\left[\tau_{k}, \text { comp }\right] \text {. }
\end{aligned}
$$

end

output global estimation $\left\{\boldsymbol{m}_{m, k}^{(i)}, \tau_{m, k}^{(i)}\right\}_{i=1}^{J_{m, k}, \text { lobal }}$.

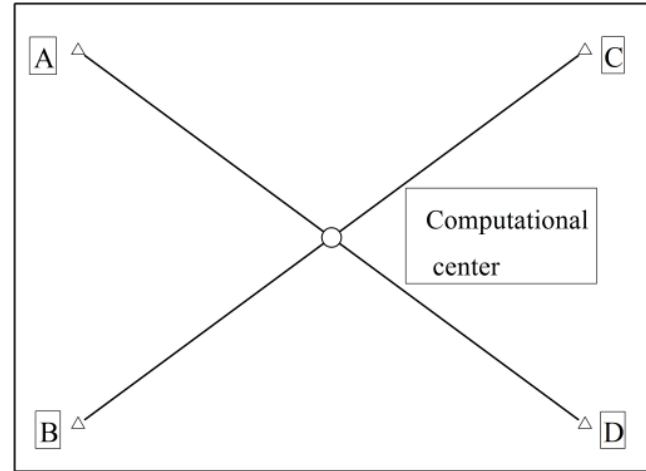

Figure 1. The Sketch Map of Network in Proposed Filter 'O'- Computational Center, ' $\Delta$ '- Node Locations

\subsection{Clutter Filter}

During implementation, we found that clutter, false measurements that the sensors may receive, can sometimes cause false alarms in global estimation. In order to improve the accuracy of distributed CK-PHD filter, we add a filter to reduce the number of clutter. The filter discards the targets exist less than 5 frames. It means that a target is considered as clutter if its surviving time is shorter than 5 frames. The procedure of clutter filter is given below.

$$
\text { input }\left\{\boldsymbol{m}_{m, k}^{(i)}, \tau_{m, k}^{(i)}\right\}_{i=1}^{J_{m, k, g l o b a l}} \text {. }
$$

Let $\hat{X}_{k-1}^{(\tau)}$ denote global estimation of the target with label $\tau$ at time $k-1, N_{\tau}$ denote the frame number of the target with label $\tau$ existing at time $k-1, \tau_{\max }$ denote the maximum element of $\hat{\tau}_{k}$, where $k=1,2, \cdots, N, N$ is the maximum of frame number.

$$
\begin{aligned}
& \text { for } \tau=1,2, \cdots, \tau_{\max } \\
& \qquad \begin{array}{c}
\text { if } !\left(\operatorname{any}\left(\tau_{k}=\tau\right)\right) \text { and }\left(N_{\tau}<5\right) \text { and }\left(N_{\tau}>0\right) \\
N_{\tau}:=0, \\
\hat{X}_{k-1}^{(\tau)}=\hat{X}_{k-2}^{(\tau)}=\cdots=\hat{X}_{k-N_{\tau}}^{(\tau)}=\phi .
\end{array}
\end{aligned}
$$

end

end 
output $\hat{X}_{k}^{(\tau)}, \tau=1,2, \cdots, \tau_{\max }$.

The output of clutter filter $\hat{X}_{k}^{(\tau)}, \tau=1,2, \cdots, \tau_{\max }$ is the estimation of distributed CKPHD filter in time $k$. The parameter $\tau$ is the tag for $\hat{X}_{k}^{(\tau)}$. The state estimations of a target can be obtained through finding $\hat{X}_{k}^{(\tau)}$ with same tag $\tau$ in different time. Finally, repeat the process to every $\hat{X}_{k}^{(\tau)}, \tau=1,2, \cdots, \tau_{\max }$, each target estimation can be obtained.

\section{Simulation Results and Discussion}

In order to validate the effectiveness of proposed algorithm, aircraft control problem, which is a classical problem in nonlinear filter, is used in simulation experiment[20]. In the scenario, there are four aircrafts which appear at 1,10,1, and 20 seconds respectively, but no spawning. Let $\boldsymbol{x}_{k}=\left[\xi_{k}, \dot{\xi}_{k}, \eta_{k}, \dot{\eta}_{k}, \Omega\right]^{\mathrm{T}}$ denote the aircraft state at time $k$, in which $\xi_{k}$ and $\eta_{k}$ are the positions of $x$ and $y$ direction, $\dot{\xi}_{k}$ and $\dot{\eta}_{k}$ are velocity of $x$ and $y$ direction, $\Omega$ is the turn rate, the system model for aircraft control problem is given by

$$
\boldsymbol{x}_{k}=\left(\begin{array}{ccccc}
1 & \frac{\sin \Omega T}{\Omega} & 0 & \frac{\cos \Omega T-1}{\Omega} & 0 \\
0 & \cos \Omega T & 0 & -\sin \Omega T & 0 \\
0 & \frac{1-\cos \Omega T}{\Omega} & 1 & \frac{\sin \Omega T}{\Omega} & 0 \\
0 & \sin \Omega T & 0 & \cos \Omega T & 0 \\
0 & 0 & 0 & 0 & 1
\end{array}\right) \boldsymbol{x}_{k-1}+\boldsymbol{v}_{k}
$$

where time interval between two adjacent measurements $T=2 \mathrm{~s}$, process noise $\boldsymbol{v}_{k}$ is satisfied with $\boldsymbol{v}_{k} \sim \mathrm{N}(\mathbf{0}, \boldsymbol{Q})$, and covariance $\boldsymbol{Q}=\operatorname{diag}\left[q_{1} \boldsymbol{M}, q_{1} \boldsymbol{M}, q_{2} T\right]$ [7]. Here,

$$
\boldsymbol{M}=\left(\begin{array}{cc}
T^{3} / 3 & T^{2} / 2 \\
T^{2} / 2 & T
\end{array}\right)
$$

Scale parameter $q_{1}$ and $q_{2}$ depend on probability density function of process noise, and set $q_{1}=0.1 \mathrm{~m}^{2} \mathrm{~s}^{-3}, q_{2}=1.75 \times 10^{-4} \mathrm{~s}^{-3}$ [7]. Here, we employ four radars, and their position coordinates are $(0,0),(15000,0),(0,-15000),(15000,-15000)$. Let $x_{0}^{(i)}$ and $y_{0}^{(i)}$ denote the $\mathrm{x}$ and $\mathrm{y}$ coordinates of the $i$ th radar, $r_{k}^{(i)}$ and $\theta_{k}^{(i)}$ denote distance and bearing measurements at time $k$, then the observation equation is given as follows

$$
\left(\begin{array}{c}
r_{k}^{(i)} \\
\theta_{k}^{(i)}
\end{array}\right)=\left(\begin{array}{c}
{\left[\left(\xi_{k}-x_{0}^{(i)}\right)^{2}+\left(\eta_{k}-y_{0}^{(i)}\right)^{2}\right]^{-1 / 2}} \\
\tan ^{-1}\left(\eta_{k} / \xi_{k}\right)
\end{array}\right)+\boldsymbol{w}_{k}
$$

where $\boldsymbol{w}_{k}$ is observation noise, which is satisfied with $\boldsymbol{w}_{k} \sim \mathrm{N}\left(\boldsymbol{0}, \boldsymbol{R}^{(i)}\right)$. Assume different radars have identical observation noise distribution, so covariance $\boldsymbol{R}^{(i)}=\operatorname{diag}\left[\sigma_{r}^{2}, \sigma_{\theta}^{2}\right]$ for $i=1,2,3,4$, besides $\sigma_{r}=10 \mathrm{~m}, \sigma_{\theta}=\sqrt{10} \times 10^{-3} \mathrm{rad}$.

The survival probability of every target in the scenario is $p_{S, k}=0.99$. Figure 2 shows the radar position and true trajectories of four targets. Figure 3 plots the changes of targets number for the true trajectories. The parameters of initial Gaussian components $\left\{w_{m, 0}^{(i)}, \boldsymbol{m}_{m, 0}^{(i)}, \boldsymbol{P}_{m, 0}^{(i)}, \tau_{m, 0}^{(i)}\right\}_{i=1}^{J_{m, 0}}$ in proposed filter are set as follows, i.e. $J_{m, 0}=2$, 
$w_{m, 0}^{(1)}=w_{m, 0}^{(2)}=0.1, \boldsymbol{m}_{m, 0}^{(1)}=[1000,300,1000,0,-3 \pi / 180]^{\mathrm{T}}, \boldsymbol{m}_{m, 0}^{(2)}=[1000,300,1000,0$, $-5 \pi / 180]^{\mathrm{T}}, \quad \boldsymbol{P}_{m, 0}^{(1)}=\boldsymbol{P}_{m, 0}^{(2)}=\operatorname{diag}\left(\left[100,10,100,10,10^{-4}\right]^{\mathrm{T}}\right), \quad \tau_{m, 0}^{(1)}=1, \quad \tau_{m, 0}^{(2)}=2$, $m=1,2,3,4$. The parameters of spontaneous births intensity $\gamma_{m, k}(\boldsymbol{x})=$ $w_{m, \gamma, k}^{(1)} \mathrm{N}\left(\boldsymbol{x} ; \boldsymbol{m}_{m, \gamma, k}^{(1)}, \boldsymbol{P}_{m, \gamma, k}^{(1)}\right) \quad$ are $\quad w_{m, \gamma, k}^{(1)}=0.1 \quad, \quad \boldsymbol{m}_{m, \gamma, k}^{(1)}=[-1000,300,-1000,0$, $-3 \pi / 180]^{T}, \boldsymbol{P}_{m, \gamma, k}^{(1)}=\operatorname{diag}\left(\left[100,10,100,10,10^{-4}\right]^{\mathrm{T}}\right)$. Corresponding tag of $\gamma_{m, k}(\boldsymbol{x})$ is $\tau_{m, \gamma, k}^{(1)}=\max \left\{\tau_{m, k-1}^{(i)}\right\}_{i=1}^{J_{m, k-1}}+1, m=1,2,3,4$.

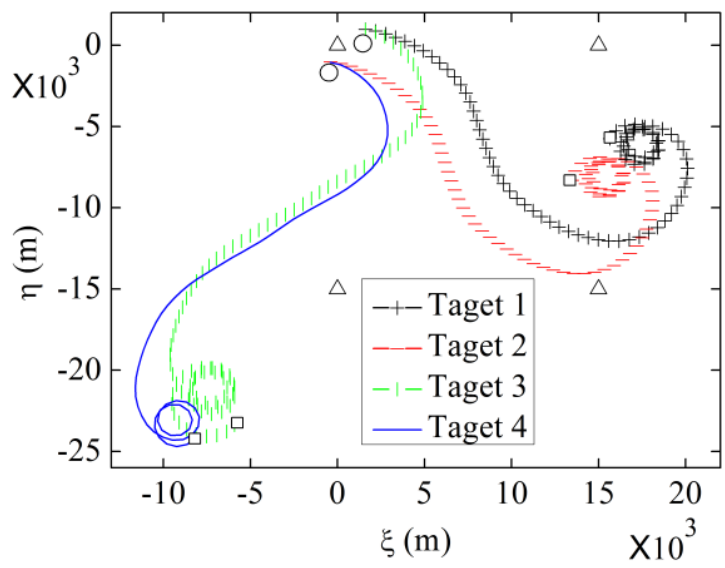

Figure 2. The Sketch Map of Targets True Trajectories. 'O'- Initial Points of Targets, ' $\square$ '- Final Points of Targets, ' $\Delta$ '- radar locations

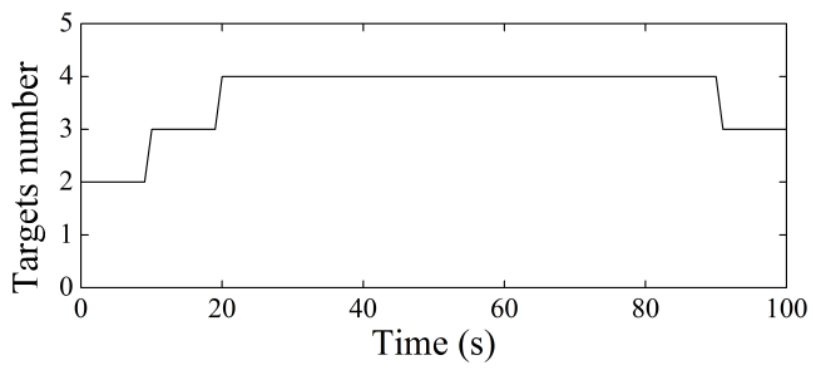

Figure 3. The Targets Number of True Trajectories with Varying Time

Every target is detected with probability $p_{D, k}=0.98$ for every radar, and the detected measurements are immersed in the clutter modeled as a Poisson RFS with intensity $\kappa_{k}(z) \triangleq \lambda_{c} \mathcal{V} u(z)$, where $\lambda_{c}=10^{-8} \mathrm{~m}^{-2}$ denotes average number of false alarm per unit area for every radar, $V=1.054 \times 10^{9} \mathrm{~m}^{2}$ denotes the area of surveillance region, $u(\cdot)$ denotes uniform density for clutter. In the simulation, merging threshold $T_{\text {merge }}$ is 4 .

In order to evaluate the performance of multiple targets tracking method effectively, two criterion, optimal subpattern assignment (OSPA) [21] and average relative error (ARE), are introduced in this paper, which can evaluate the accuracy of targets states and number. Let $X=\left\{\boldsymbol{x}_{1}, \boldsymbol{x}_{2}, \cdots, \boldsymbol{x}_{|X|}\right\}$ denote true targets states, $\hat{X}=\left\{\hat{\boldsymbol{x}}_{1}, \cdots, \hat{\boldsymbol{x}}_{|\hat{X}|}\right\}$ denote estimations of targets states, $\Pi_{|\hat{X}|}$ denote the set of permutations on $\{1,2, \cdots,|\hat{X}|\}$ with the cardinality $|X|$. Therefore, $p$ th-order OSPA with $|X| \leq|\hat{X}|$ is formed by 


$$
\bar{d}_{p}^{(n)}(X, \hat{X})=\left(\frac{1}{|\hat{X}|}\left(\min _{\pi \in \Pi_{|\hat{X}|}} \sum_{i=1}^{|X|} d^{(n)}\left(\boldsymbol{x}_{i}, \hat{\boldsymbol{x}}_{\pi(i)}\right)^{p}+(|\hat{X} H X|) n^{p}\right)\right)^{1 / p}
$$

if $|X|>|\hat{X}|, \bar{d}_{p}^{(n)}(X, \hat{X})=\bar{d}_{p}^{(n)}(\hat{X}, X)$. For criticizing the algorithm accuracy of estimating targets number, average relative error (ARE) is defined by

$$
\mathrm{ARE}=\mathrm{E}\left\{\frac{\|\hat{X}|-| X\|}{|X|}\right\}
$$

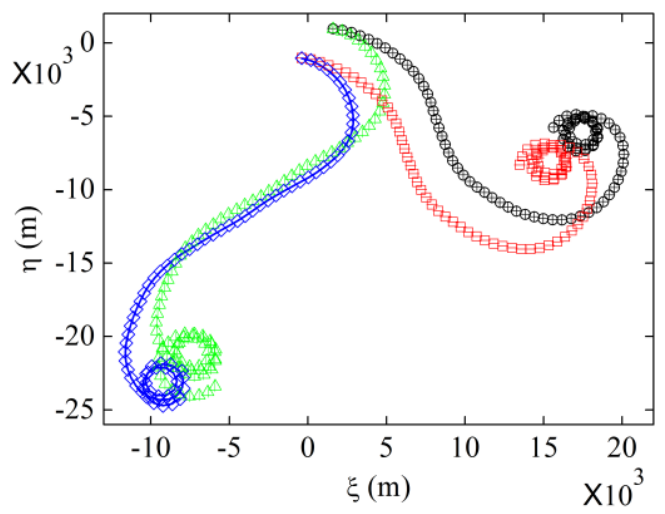

Figure 4. Estimates of Targets' Position with Proposed Filter. '+'- True Trajectory of Target 1, '-'- True Trajectory of Target 2, '|'- True Trajectory of Target 3, '---'- True Trajectory of Target 4, 'O'- Estimates of Target 1, ' $\square$ '-

\section{Estimates of Target 2, ' $\Delta$ '- Estimates of Target 3, ' $\diamond$ '- Estimates of Target 4}

For evaluating the performance of target tracking filter, we compared proposed algorithm's result with performance of EK-PHD, UK-PHD, CK-PHD. Performance of GM-PHD tracker which tag management algorithm [19] was proposed in was not compared in this paper as the tracker apply in linear system while simulation experiment is a nonlinear problem. Distributed multi-target tracking using JPDA [6] is not used in comparison experiment as well because it is only suitable for targets tracking where targets' number is given in advance while the targets' number is mutative and unknown in our simulation experiment. Note that in order to be identical as corresponding literature of EK-PHD [10], UK-PHD [10], and CK-PHD [13] filter, tag management is not included in their implementation for comparison. Figure 4 shows estimations of targets' positions with proposed filter, in which the estimations are obtained via 100 Monte Carlo simulations. From Figure 4, we can see that proposed filter can not only track targets but also distinguish the targets, which cannot be realized using EK-PHD, UK-PHD and CK-PHD filter. Performance comparisons of four algorithms are shown from Figure 5 to Figure 8.

Figure 5 shows the second-order OSPA and ARE result of these four filters via 100 Monte Carlo simulation, where the OSPA criterion is two-order. Observe that the results of four filters are almost identical especially for ARE when time is earlier than 10, however, proposed filter always has better estimation result at other time.

In order to analyze the robustness of proposed filter, the performance of the four filters are compared when detection probability $p_{D, k}$, average number of false alarm per unit area for every radar $\lambda_{c}$ and time interval between two adjacent measurements $T$ vary respectively. 


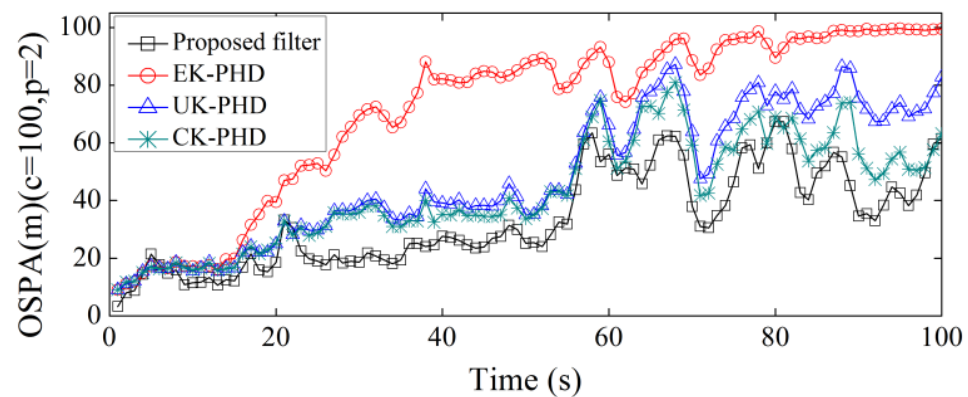

(a)

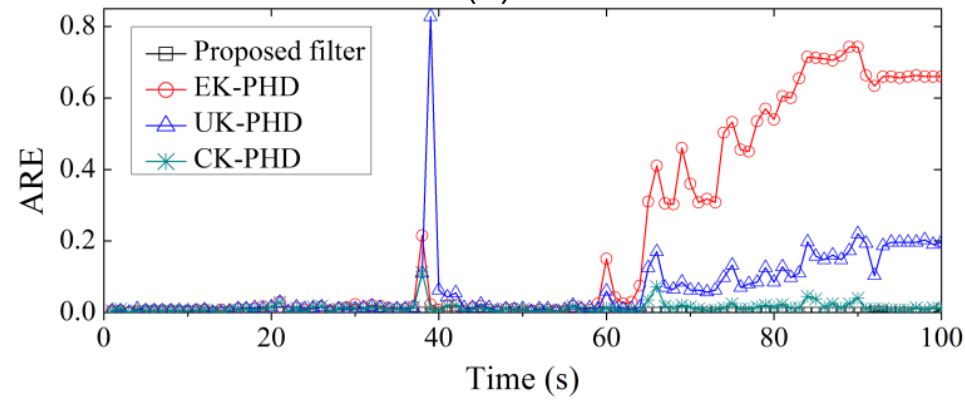

(b)

Figure 5. OSPA and ARE Comparison of EK-PHD, UK-PHD, CK-PHD and Proposed Filter

Figure 6 shows the OSPA and ARE results of four filters against various $p_{D, k}$ with other parameters fixed at $\lambda_{c}=10^{-8}, T=2 \mathrm{~s}$. Here, ARE results of CK-PHD and proposed filter in Figure 6 (b) are so close to be distinguished easily, therefore Figure 6 (c) shows ARE results of CK-PHD and proposed filter. Observe that the change of $p_{D, k}$ have an effect on the performance of EK-PHD and UK-PHD, but almost no influence on the filter results of CK-PHD and proposed filter. CK-PHD and proposed filter can estimate nonlinear motion more accurately due to the feature that components representing targets have higher weights in CK-PHD and proposed filter than in other two algorithms. This feature also ensures that variation of $p_{D, k}$ which affects weights of Gaussian components has little influence on states estimation in CK-PHD and proposed filter. Hence these two filters have better results and are almost not affected by $p_{D, k}$ changing. Furthermore, performance of proposed filter is superior to CK-PHD's from Figure 6 (a) and Figure 6 (c).

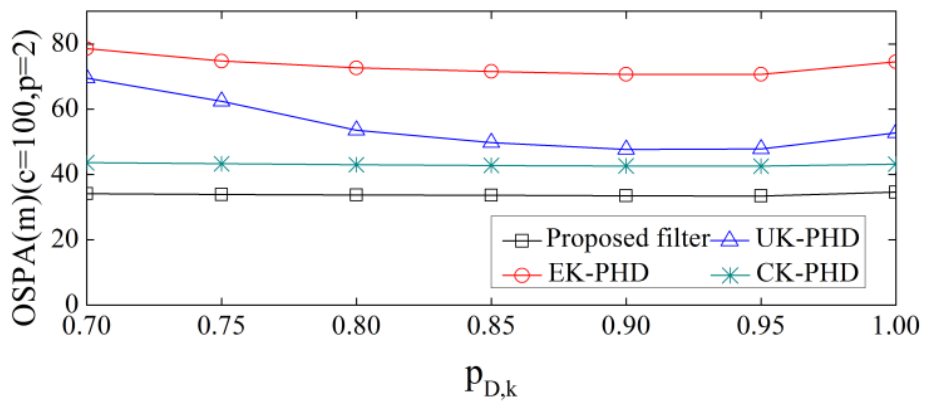

(a) 


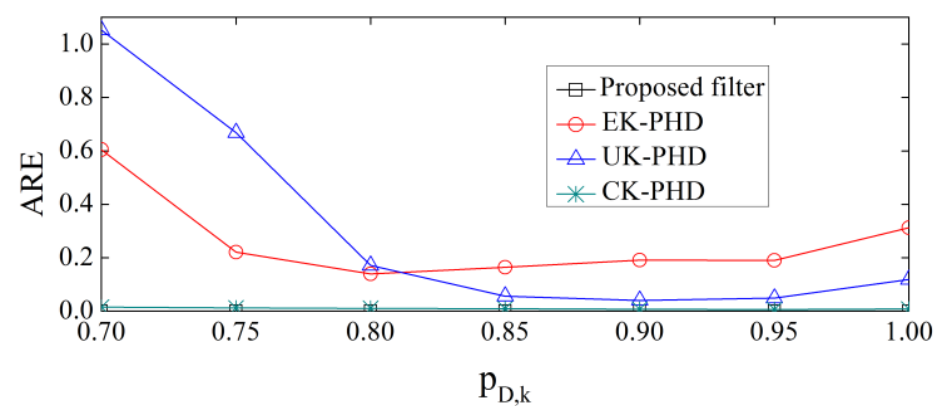

(b)

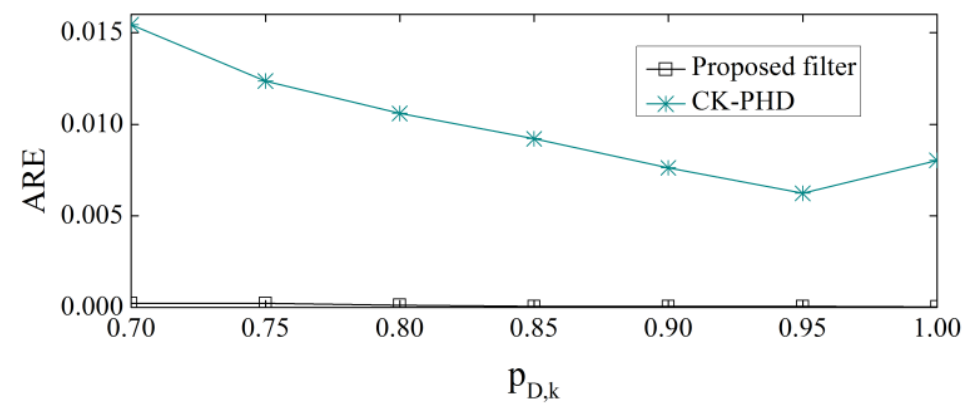

(c)

Figure 6. OSPA and ARE Comparison of EK-PHD, UK-PHD, CK-PHD and Proposed Filter with Varying $p_{D, k}$

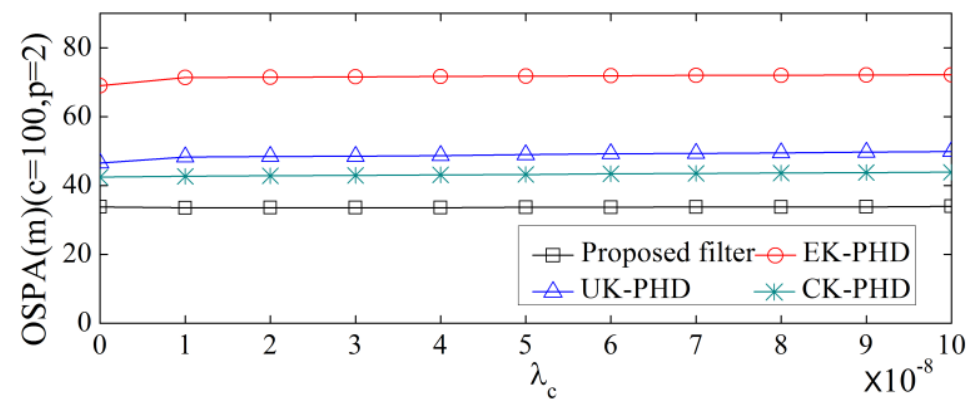

(a)

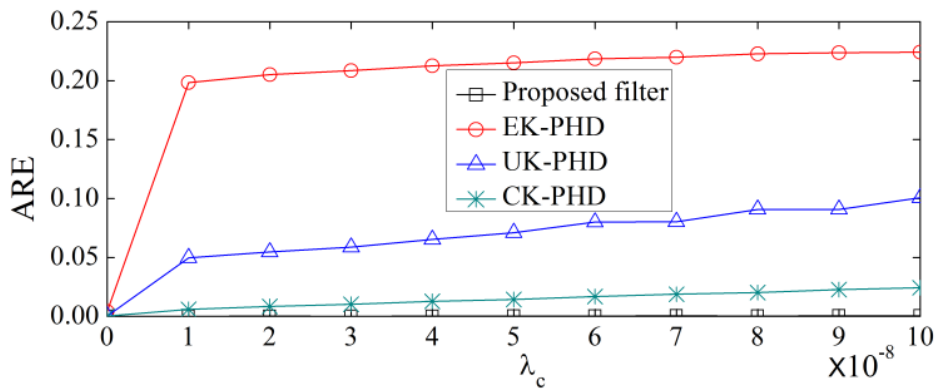

(b)

Figure 7. OSPA and ARE Comparison of EK-PHD, UK-PHD, CK-PHD and Proposed Filter with Varying $\lambda_{c}$

Figure 7 shows the OSPA and ARE results of four filters against various $\lambda_{c}$ with other parameters fixed at $p_{D, k}=0.98, T=2 \mathrm{~s}$. It can be seen that the estimations of targets number in four filters are more accurate when $\lambda_{c}=0$, that is, no false alarm in the 
surveillance area. With $\lambda_{c}$ increasing, false alarm number grows up, and the results especially ARE rise. However, proposed filter has smaller OSPA and ARE results than other filters.

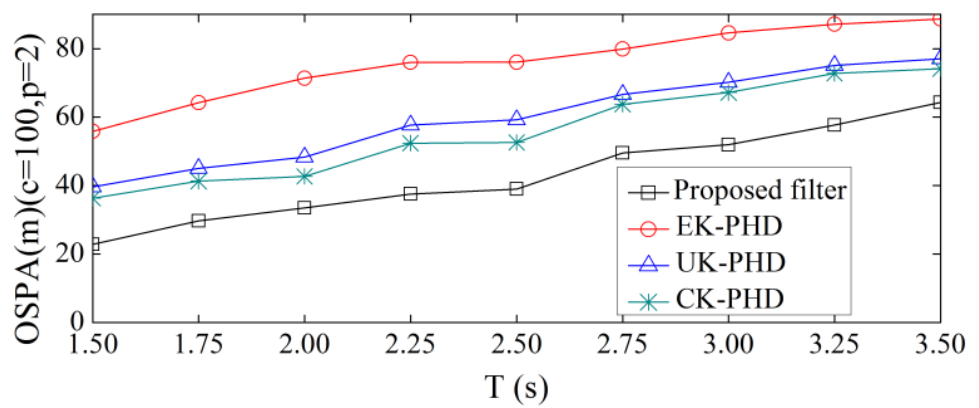

(a)

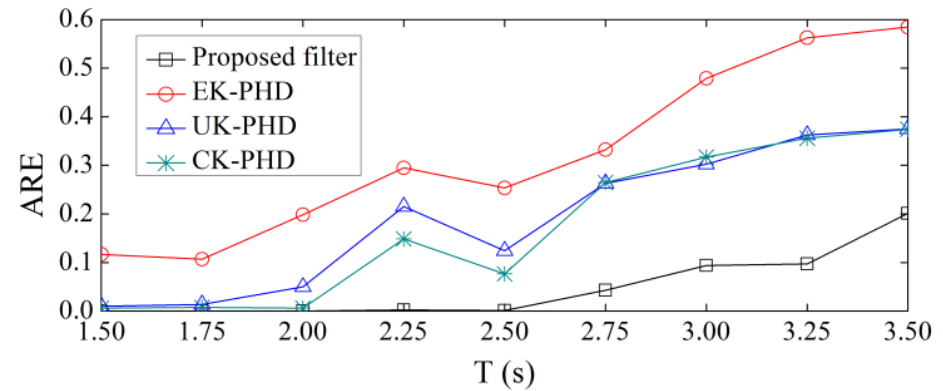

(b)

Figure 8. OSPA and ARE Comparison of EK-PHD, UK-PHD, CK-PHD and Proposed Filter with Varying $T$

Figure 8 shows the OSPA and ARE results of four filters against time interval between two adjacent measurements $T$ with other parameters fixed at $p_{D, k}=0.98, \lambda_{c}=10^{-8}$. Figure 8 shows that proposed filter has smaller OSPA and ARE results than EK-PHD, UK-PHD and CK-PHD filter when $T$ changes from $1.5 \mathrm{~s}$ to $3.5 \mathrm{~s}$, which means the proposed filter is better while time interval between two adjacent measurements changes.

The simulation results of Figure 5 - Figure 8 indicate that proposed filter has better tracking performance than EK-PHD, UK-PHD and CK-PHD filter, even detection probability changes, average number of false alarm per unit area for every radar increases, time interval between two adjacent measurements varies.

Based on the system model (15) and (17), computational complexity comparison of EK-PHD, UK-PHD, CK-PHD and proposed filters is given for analysis. Let $J_{k-1}$ and $J_{\gamma, k}$ denote Gaussian component numbers of probability hypothesis density $v_{k-1}$ and spontaneous births probability hypothesis density $\gamma_{k}$ respectively, $J_{\beta, k}$ denote spawned targets number, $n_{x}$ and $n_{z}$ denote the dimension of target state and measurement respectively, $M$ denote the sensors number, $\left|\boldsymbol{Z}_{k}\right|$ denote the number of measurements for one sensor. Take PHD recursion for example, Table 1 shows the computational complexity comparison of four filters, where $J=J_{\gamma, k}+J_{k-1} J_{\beta, k}$. It can be seen that the computational complexity of CK-PHD filter is a little higher than EK-PHD and UK-PHD. Because measurements that are used in proposed filter are obtained from $\mathrm{M}$ sensors, computational complexity of proposed algorithm is M times of CK-PHD. 
Table 1. Computational Complexity Comparisons of EK-PHD, UK-PHD, CKPHD and Proposed Filters

\begin{tabular}{|c|c|c|}
\hline & $\begin{array}{c}\text { Addition, subtraction and } \\
\text { multiplication operations }\end{array}$ & $\begin{array}{c}\text { Nonlinear operations } \\
\text { (sine and root) }\end{array}$ \\
\hline EK-PHD & $o\left(\left(n_{z}^{2}+n_{x}^{3}+n_{x}^{2} n_{z}\right)\left|\boldsymbol{Z}_{k}\right| J\right)$ & $o\left(\left(2\left|\boldsymbol{Z}_{k}\right|+0.2 n_{x} n_{z}\right) J\right)$ \\
\hline UK-PHD & $o\left(\left(6 n_{x} n_{z}^{2}+n_{z}^{3}+n_{x}^{2}\left|\boldsymbol{Z}_{k}\right|\right) J\right)$ & $o\left(\left(2 n_{x} n_{z}\right) J\right)$ \\
\hline CK-PHD & $o\left(\left(7 n_{x} n_{z}^{2}+\left|\boldsymbol{Z}_{k}\right| n_{x}^{2}+3 n_{x}^{3}+\left|\boldsymbol{Z}_{k}\right| n_{x} n_{z}\right) J\right)$ & $o\left(0.5 n_{x}^{3} J_{k-1}+2 n_{x} n_{z} J\right)$ \\
\hline $\begin{array}{c}\text { Proposed } \\
\text { filter }\end{array}$ & $o\left(\left(7 n_{x} n_{z}^{2}+\left|\boldsymbol{Z}_{k}\right| n_{x}^{2}+3 n_{x}^{3}+\left|\boldsymbol{Z}_{k}\right| n_{x} n_{z}\right) J M\right)$ & $o\left(\left(0.5 n_{x}^{3} J_{k-1}+2 n_{x} n_{z} J\right) M\right)$ \\
\hline
\end{tabular}

\section{Conclusions}

Aiming at distributed multi-target tracking problem, this paper proposes a distributed cubature Kalman-probability hypothesis density filter for tracking multiple targets. This method uses the measurements from multiple sensors in the scenario, and is applied to multi-target tracking system without the assumption that targets' appearance and disappearance time are certain and targets' number is pre-known. Moreover, this method does not need to calculate Jacobian matrix or to set scaling parameters. Simulation results show that the proposed method improves robustness enhances the estimation accuracy of targets number and states. We can conclude that the proposed method is an effective solution to distributed multi-target tracking problem in nonlinear Gaussian system.

\section{Acknowledgments}

This work was supported by the National Natural Science Foundation of China (No. 61403060), Independent Research Fund of Dalian Minzu University (No.DC201501061 and DCPY20160003), Dalian Science and Technology Planning Project (No.2015A11GX021) and the Doctoral Scientific Research Foundation of Liaoning Province (No.201601017).

\section{References}

[1] E. Fan, S. Shen, K. Hu, C. Yuan and P. Wang, "Uncertainty Analysis of Multiple Target Tracking in Distributed Sensor Networks", International Journal of Control and Automation, vol. 9, no.6, (2016), pp.127-136.

[2] A. T. Kamal, J. A. Farrell and A. K. Roy-Chowdhury, "Information consensus for distributed multitarget tracking", Conference on Computer Vision and Pattern Recognition, Portland, USA, (2013).

[3] T. Y. Rezaii and M.-A. Tinati, "Distributed multi-target tracking using joint probabilistic data association and average consensus filter", Annals of Telecommunications, vol. 66, (2011), pp.553-566.

[4] D. Smith and S. Singh, "Approaches to multisensor data fusion in target tracking: a survey", IEEE Transactions on Knowledge and Data Engineering, vol.18, no.12, (2006), pp.1696-1710.

[5] R. Mahler, "Multitarget Bayes filtering via first-order multitarget moments", IEEE Transactions on Aerospace and Electronic Systems, vol.39, no.4, (2003), pp.1152-1178.

[6] R. Mahler, "A theoretical foundation for the Stein-Winter probability hyphthesis density (PHD) multitarget tracking approach", Proceedings of the MSS National Symposium Sensor Data Fusion, San Antonoi, USA, (2000).

[7] I. Arasaratnam and S. Haykin, "Cubature Kalman filter", IEEE Transactions on Automatic Control, vol.54, no.6, (2009), pp.1254-1269.

[8] J. Yin, J. Zhang and Z. Zhuang, "Gaussian sum PHD filtering algorithm for nonlinear non-Gaussian models", Chinese Journal of Aeronautics, vol.21, (2008), pp.341-351.

[9] B-N. Vo and S. Singh, "Sequential Monte Carlo methods for multitarget filtering with random finite sets", IEEE Transactions on Aerospace and Electronic Systems, vol.41, no.4, (2005), pp.1224-1245.

[10] B. Vo and W. Ma, "The Gaussian mixture probability hypothesis density filter", IEEE Transactions on Signal Processing, vol.54, no.11, (2006), pp.4091-4014.

[11] L. Chen, Z. Chen, F. Yin and D. Hou, "Central difference Kalman-probability hypothesis density filter for multi-target tracking", Control and Decision, vol.28, no.1, (2013), pp.36-42.

[12] L. Chen, Y. Zhang and W. Yu, "Central Difference Kalman-probability Hypothesis Density Filter for 
Multiple Speakers Tracking", Journal of Information and Computational Science, vol.11, no.8, (2014), pp.2735-2746.

[13] D. Macagnano and G. T. F. De Aberu, "Multitarget tracking with the cubature Kalman probability hypothesis density filter", Conference of the Forth Fourth Asilomar Conference on Signals, Systems and Computers, Pacific Grove, USA, (2010).

[14] O. Hlinka, F. Hlawatsch and P.M. Djurić, "Distributed particle filtering in agent networks", IEEE Signal Processing Magazine, vol.1, (2013), pp.61-81.

[15] R. Olfati-Saber, "Distributed Kalman filter with embedded consensus filters", Proceedings of the 44th IEEE Conference on Decision and Control and the European Control Conference, Seville, Spain, (2005).

[16] W. Li and Y. Jia, "Consensus-based distributed multiple model UKF for jump Markov nonlinear systems", IEEE Transactions on Automatic Control, vol.57, no.1, (2012), pp.230-236.

[17] Y. Sheng, X. Hu and P. Ramanathan, "Distributed particle filter with GMM approximation for multiple targets localization and tracking in wireless sensor network", Proceeding of the 4th International Symposium on Information Processing in Sensor Networks, Los Angeles, USA, (2005).

[18] X. R. Li and V. P. Jilkov, "Survey of maneuvering target tracking Part I: dynamic models", IEEE Transactions on Aerospace and Electronic Systems, vol.39, no.4, (2003), pp.1333-1364.

[19] K. Panta, D.E. Clark and B-N. Vo, "Data association and track management for the Gaussian mixture probability hypothesis density filter", IEEE Transactions on Aerospace and Electronic Systems, vol.45, no.3, (2009), pp.1003-1016.

[20] X. R. Li and V. P. Jilkov, "Survey of maneuvering target tracking Part I: dynamic models", IEEE Transactions on Aerospace and Electronic Systems, vol.39, no.4, (2003), pp.1333-1364.

[21] D. Schuhmacher, B-T. Vo and B-N. Vo, "A consistent metric for performance evaluation of multi-object filters”, IEEE Transactions on Signal Processing, vol.56, no.8, (2008), pp.3447-3457.

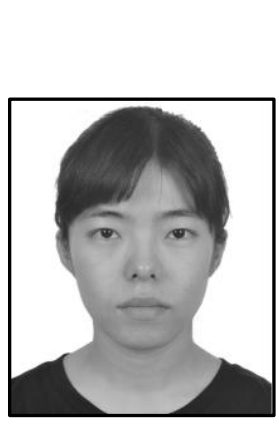

Authors

Liming Chen, she received her B.S. degree in electronic engineering from Jinlin University, Changchun, China, in 2003, and the M.S. degree in measuring and testing technology and instruments and the Ph.D. degree in signal and information processing from Dalian University of Technology, Dalian, China, in 2006 and 2013 respectively. She joined the Department of Electronic Engineering, Dalian Minzu University, as a Lecture in 2013. Her research interests include multi-target tracking and speech processing.

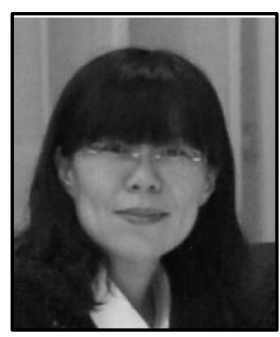

Mingxin Jiang, she received the B.S. degree and the M.S. degree from Jilin University, Changchun, China, in 2002 and 2005. She received a Ph.D. degree in Signal and information processing, Dalian University of Technology, China, in 2013. She is currently an associate professor in Faculty of Computer and Software Engineering at Huaiyin Institute of Technology. Her research interests include multi-object tracking and vision sensors for robotics. 
International Journal of Control and Automation

Vol. 10, No. 11 (2017) 